\title{
Rehabilitación de paciente fumador con prótesis hibrida sobre seis implantes: Reporte de caso
}

\author{
Smoking patient rehabilitation with hybrid prosthesis on six implants: Case report
}

Carmen Eliana López López ${ }^{1, a}$, Martin Quintana del Solar ${ }^{1, b}$

\section{RESUMEN}

La prótesis sobre implantes es una buena alternativa de tratamiento frente a los tratamientos convencionales de prótesis total en pacientes con edentulismo total. Este tratamiento se ha venido realizando con éxito en los últimos años y constituye una realidad clínica de alto valor. El presente artículo reporta el caso clínico de un paciente de sexo masculino fumador donde se realizó la rehabilitación del maxilar superior con una prótesis tipo hibrida sobre 6 implantes. A continuación se describe paso a paso la elaboración de una prótesis implanto soportada, devolviéndole al paciente estética y funcionabilidad mejorando su calidad de vida..

PALABRAS CLAVE: Prótesis sobre implantes, prótesis hibrida, prótesis implanto soportada.

\section{SUMMARY}

The oral rehabilitation with implant prosthesis has been incorporated into dental practice as an alternative to conventional treatment in patients with complete edentulous. This treatment has been carried out successfully in recent years and is a clinical reality of high value. This article reports the case of a male patient with a history of being a smoker in which the rehabilitation of the upper jaw was restored with a hybrid type prosthesis on 6 implants. The following describes step by step the elaboration of implant-supported prosthesis, restoring the aesthetics and functionality thereby improving patient quality of life.

KEYWORDS: Implant prosthetics, hybrid prosthetics, implant supported prosthesis. 


\section{INTRODUCCION}

La rehabilitación oral mediante el tratamiento con implantes es una alternativa de tratamiento queviene siendo muy utilizada en los últimos años. Las opciones protésicas para rehabilitar un paciente completamente edéntulo con implantes dentales se dividen en: restauraciones fijas y restauraciones removibles (1).

La prótesis hibrida consiste en una estructura metálica colada recubierta por acrílico y que a su vez soporta dientes fijos artificiales. El diseño original de la prótesis hibrida (fija-removible) fue desarrollado por investigadores suecos utilizando el sistema de implante intraóseo de dos etapas desarrollado por Branemark. La prótesis consistió en una estructura de aleación de oro unido a las cofias de los implantes, y sobre esta dientes de acrílico de dentadura convencional asegurados con resina acrílica (2).

Los factores que determinan el tipo de restauración sobre implantes en un paciente edéntulo total es la cantidad de espacio desde el hueso hasta el plano oclusal (espacio protésico) y el soporte labial. El espacio protésico que se necesita para una prótesis hibrida es de $11 \mathrm{~mm}$ como mínimo y como máximo $15 \mathrm{~mm}$, existiendo un soporte labial dado por las mismas estructuras óseas. Cuando se dispone de menos espacio $(10 \mathrm{~mm})$ y existe soporte labial, se sugiere la restauración fija de metal porcelana sobre implantes; cuando existe más de 15 mmde espacio protésico y ausencia de soporte labial se sugiere una restauración tipo sobredentadura sobre implantes la cual dará ese soporte labial que no la dan las estructuras óseas del paciente (1).

Zarb y col. describen el tratamiento en maxilas edéntulas totales severamente reabsorbidas con una prótesis híbrida utilizando una estructura metálica con acrílico y dientes artificiales, con espacios prótesis mayores a $15 \mathrm{~mm}(3)$.

La desadaptación entre la estructura metálica y los implantes puede causar perdida de hueso y fracaso en la oseointegración, clínicamente esto es determinante. Generalmente es aceptado por la literatura que el asentamiento pasivo de una prótesis es requerida para el mantenimiento y éxito a largo plazo para un tratamiento sobre implantes. Además la literatura ha implicado que la desadaptación de la estructura metálica es un factor determinante y significativo en las causas de complicaciones mecánicas y biológicas. El aflojamiento de tornillos tanto de la prótesis como del pilar e incluso la fractura de varios componentes del sistema ha sido atribuido a la falta de ajuste y adaptación de la prótesis. Adicionalmente, reacciones adversas de los tejidos, dolor, sensibilidad, pérdida de hueso marginal y pérdida de oseointegración son también una posible lista de secuela de la falta de adaptación de la prótesis (4).

El consumo de tabaco y el hábito de fumar no es una contraindicación para la colocación de implantes dentales, sin embargo el fumar puede perjudicar la osteointegración, al comprometer el hueso que rodea al implante. La cantidad de tabaco también influye indirectamente en la vida media del implante, haciendo que esta se vea reducida cuando se compara con un paciente no fumador (5).

Los pacientes fumadores tienen mayor riesgo de complicaciones que los pacientes no fumadores, aunque la terapia implantologica se puede realizar motivando a los pacientes fumadores a disminuir o cesar el hábito de fumar (5). Los estudios demuestran tasas de éxito más bajas para los implantes dentales en pacientes fumadores. Bain y Moy realizaron un estudio retrospectivo con 2194 implantes colocados a lo largo de 7 años, el tabaco fue el más importante factor de riesgo en la pérdida de los implantes. Los resultados mostraron de forma significativa que la tasa de fracasos era del $4,7 \%$ en los no fumadores, frente al $11,3 \%$ en los fumadores (6).

Se ha visto que dejar de fumar postcirugía mejora la supervivencia del implante. La utilización de nuevas superficies en los implantes dentales parece mejorar las tasas de éxito entre los pacientes fumadores. En un estudio realizado en 1999 por Grunder se vio un éxito del 98,6\% a los 34 meses, sin diferencias entre los fumadores y no fumadores. De hecho, dejar de fumar mejora las expectativas de éxito de los implantes entre los pacientes fumadores y esto lo iguala a la de los pacientes no fumadores (7).

Idealmente, el paciente debe dejar de fumar 2 semanas antes de la colocación de implantes, esto mejora la circulación sanguínea y la agregación plaquetaria. Luego debe dejar de fumar 8 semanas despues, donde ocurre la fase osteoblastica, esto aumenta la cicatriza- 
ción e invierte la microflora (8).

Es importante que los pacientes sepan los riesgos del fumar y de la cantidad de tabaco que consumen. El consumo de tabaco o el hábito de fumar no representa una contraindicación para colocar implantes dentales pero aumenta el riesgo de pérdida y la no osteointegración así como disminuye su supervivencia (7).

El propósito de este artículo fue presentar el reporte de un caso clínico de un paciente fumador, edéntulo total superior, que recibió tratamiento con implantes y la instalación de una prótesis hibrida, mostrando el paso a paso de la confección de dicha prótesis. dicha prótesis.

\section{CASO CLINICO}

\section{Fase de diagnostico}

Paciente de sexo masculino de 59 años de edad, fumador (12 cigarrillos al día), con enfisema pulmonar, acudió al Servicio del Postgrado de Rehabilitación Oral de la Facultad de Estomatología de la Universidad Peruana Cayetano Heredia (UPCH). Al examen clínico extraoral presentó cráneo mesocéfalo, cara mesofacial, normodivergente y perfil ligeramente convexo (Figura 1 y figura 2).

En cuanto a sus antecedentes estomatológicos refirió haber perdido dientes por caries y enfermedad periodontal, presento dificultad para masticar por falta de dientes. Se colocó 5 implantes en maxilar superior en

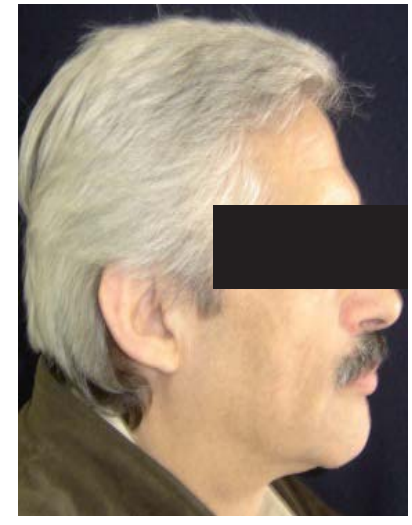

Figura 1. Vista lateral

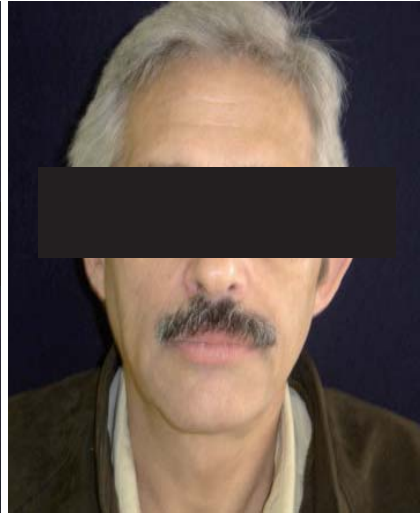

Figura 2. Vista frontal octubre del 2009 en una consulta privada con pérdida de 3 implantes a la semana de la cirugía.Al examen clínico intraoral presentóencía eritematosa, lisa y de consistencia edematosa, biotipo periodontal grueso (tipo II), frenillo antero superior en encía adherida, reabsorción horizontal y vertical de rebordes edéntulos. Presentó 8 dientes en boca 2 en el maxilar superior y seis en el maxilar inferior. Fue portador de una prótesis parcial removible superior y de prótesis fija inferior ambos en mal estado (Figura 3 y figura 4).

El diagnóstico del paciente fue en los tejidos periodontales de periodontitis crónica generalizada severa. Condiciones mucogingivales alrededor del reborde edéntulo presento deficiencia en altura/ancho: Seibert

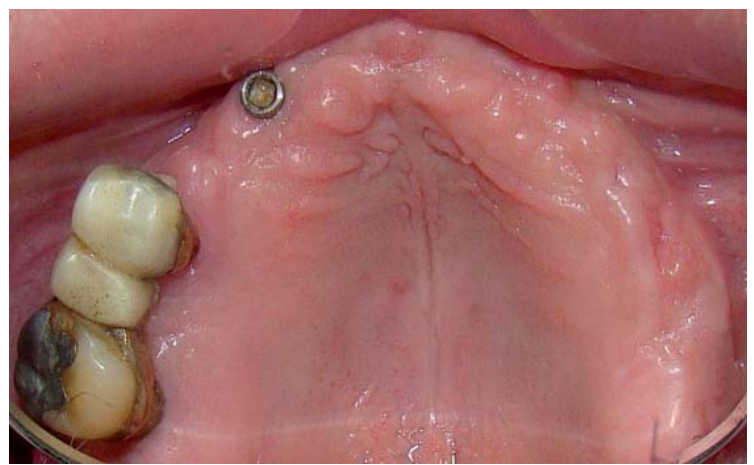

Figura 3. Maxilar superior

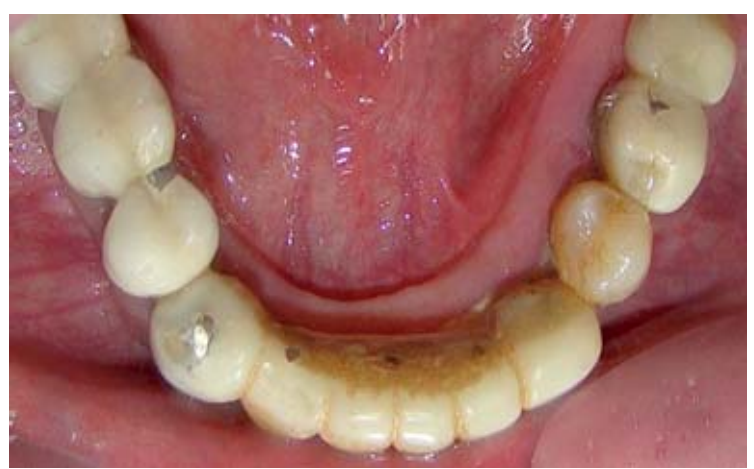

Figura 4. Maxilar Inferior

III y trauma de oclusión secundario. En cuanto a la oclusión presentó Disarmonía Oclusalcon edéntulismo parcial superior e inferior, alteración de la guía anterior, alteración del plano oclusal y alteración de la dimensión vertical (Figura 5,6 y 7). Se tomó una radiografía panorámica para evaluar las estructuras óseas y dentarias (Figura 8).

Se planifico inicialmente controlar y mejorar las condiciones de salud gingival, restablecer la guía anterior y estabilizar la oclusión para corregir el desorden 


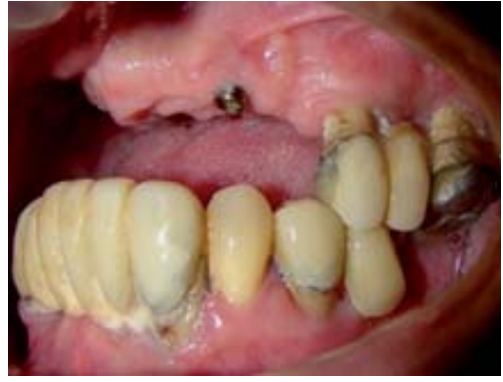

Figura 5. Vista Lateral Der.

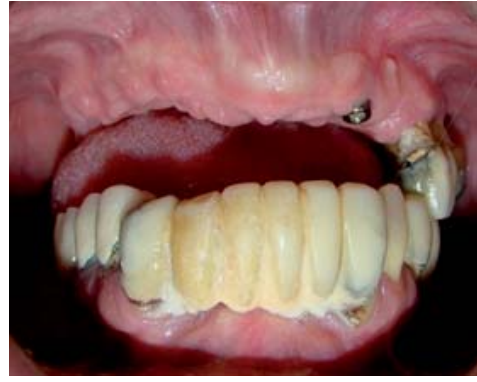

Figura 6. Vista frontal

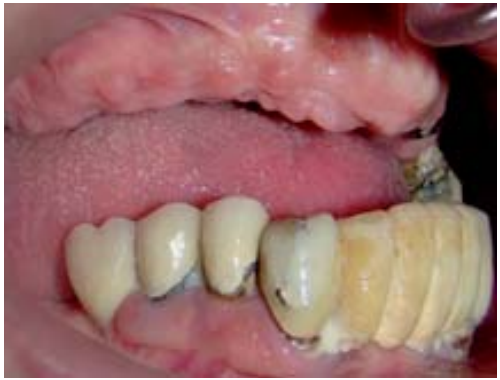

Figura 7. Vista Lateral Izq.

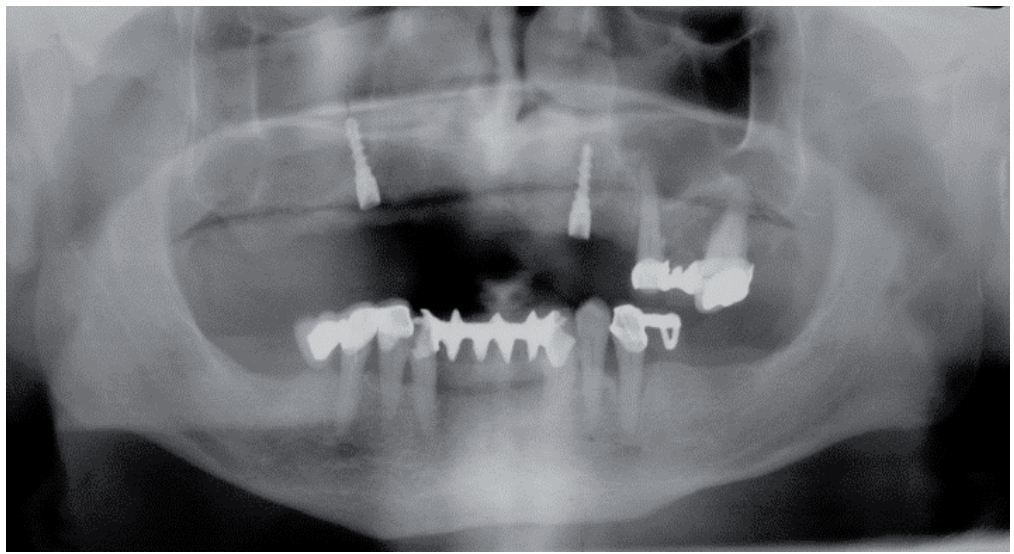

Figura 8. Radiografía panorámica inicial

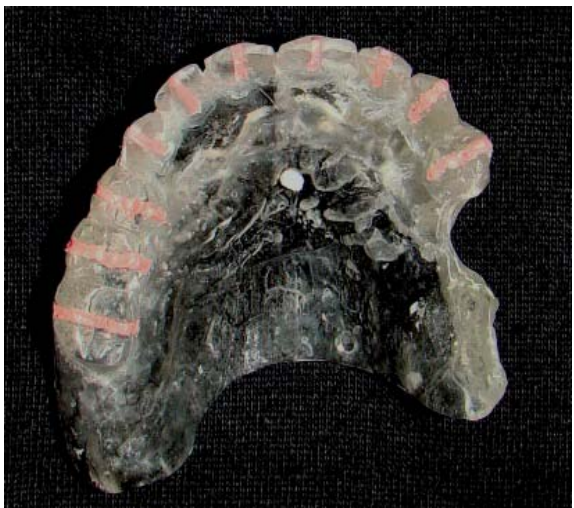

Figura 9. Guía tomográfica.

oclusal mediante una prótesis provisional. Se tomaron impresiones y los modelos fueron montados en articulador semiajustable para la confección de una prótesis parcial acrílica que sirvió de encerado diagnóstico y duplicado en acrílico transparente de termocurado para ser usado como guía tomografía(Figura 9). La guía tomografía fue probada en boca del paciente (Figura 10). Mediante el programa Dental Slice se planificó la colocación de 6 implantes superiores (Figura11).

\section{Fase quirúrgica}

El paciente fue derivado al servicio del postgrado de Periodoncia e Implantes de la UPCH y se procedió a dar tratamiento periodontal con exodoncias de las piezas 2.5 y 2.7, retiro de los implantes a nivel de las piezas 1.4 y 2.3. Posteriormente se colocó en diciembre del 2010 los 6 implantes en el maxilar superior según lo planificado (Figura 12). Luego de la cirugía se instaló una prótesis total inmediata rebasada con acondicionador de tejido, y en el maxilar inferior diez 


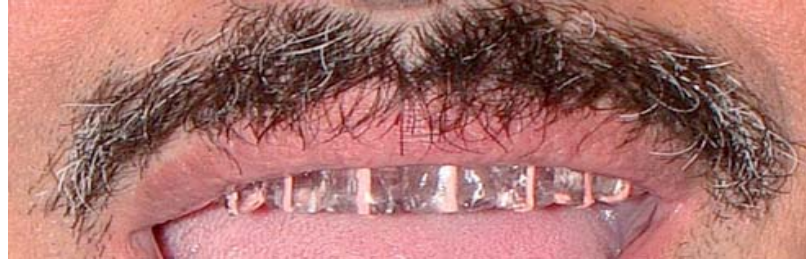

Figura 10. Prueba en boca de guía tomográfica.

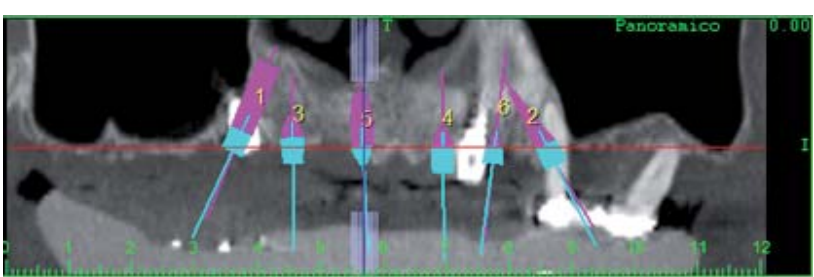

Figura 11. Planificación mediante Dental Slice.

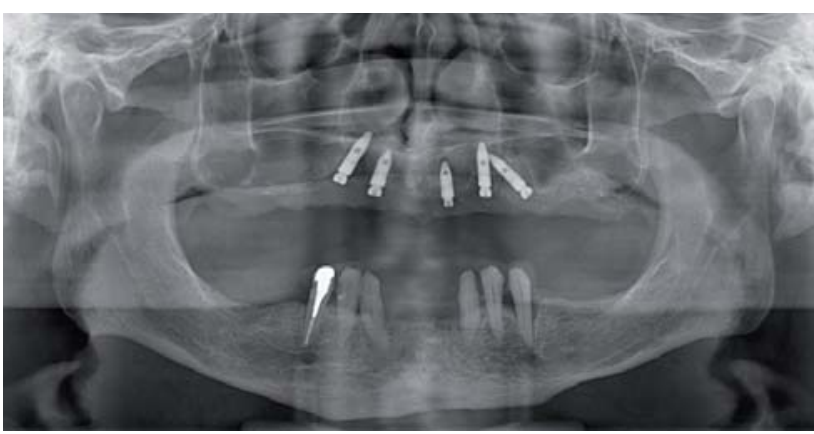

Figura 12. Radiografía panorámica post colocación de implantes.

coronas de termocurado para estabilizar la oclusión.

\section{Fase protésica y fase de mantenimiento}

Pasado el periodo de oseointegración de 6 meses, se procediócon la cirugía de segunda fase de tratamiento con la exposición de las plataformas de los implantesy a la selección de los pilares midiendo la profundidad del transmucoso con ayuda del probador de pilares, se usaron seis Mini Pilares CMNeodent ${ }^{\circledR}$, de los cuales cuatro tuvieron una angulación de $30^{\circ}$, uno de $17^{\circ}$ y uno recto (Figuras 13 y 14).Los cuales fueron ajustados con un torque de $15 \mathrm{~N}$ para los pilares angulados y de $30 \mathrm{~N}$ para el pilar recto (Según las indicaciones del fabricante).

Posteriormente se procedió a la transferencia de la

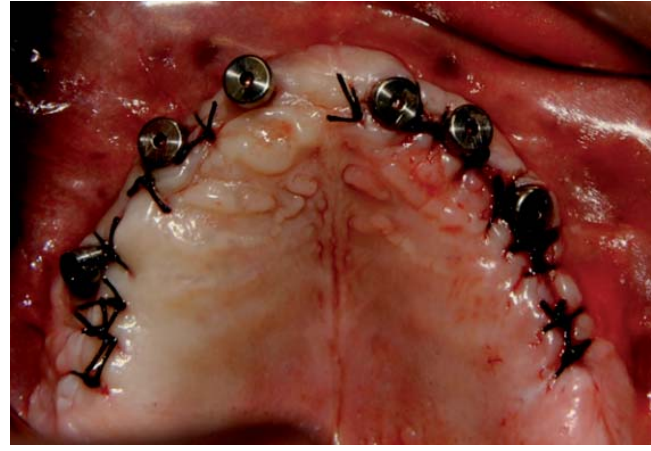

Figura 13. Cirugía de segunda fase.

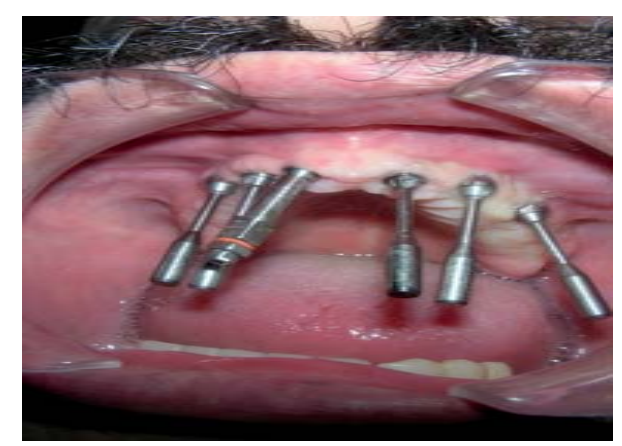

Figura 14. Selección de pilares

posición de los pilares con el uso de aditamentos protésicos (transfer) estos fueron ferulizados con acrílico de combustión completa (Duralay®),se colocaron los análogos de los pilares y se insertaron en un bloque de yeso para confeccionar una ferulización de los transfer con acrílico de termocurado, tratando de esta manera de reducir al máximo la contracción del metal de la futura estructura colada y así obtener un asentamiento pasivo de esta (Figuras 15, 16 y 17).

Esta estructura fue colocada sobre los implantes y se

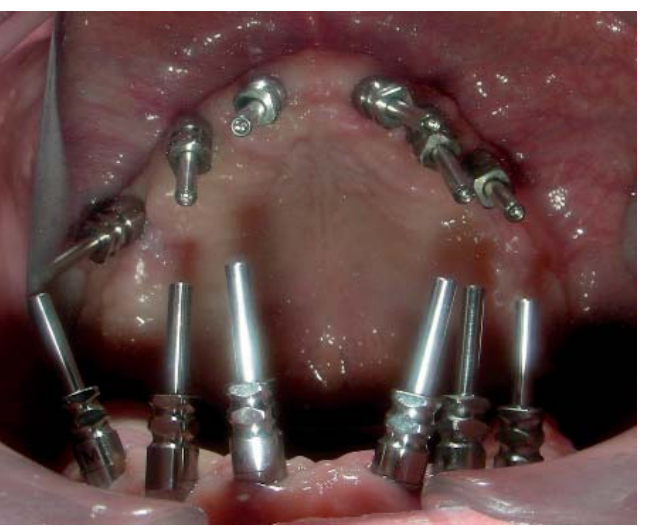

Figura 15. Transfers ubicados en boca. 


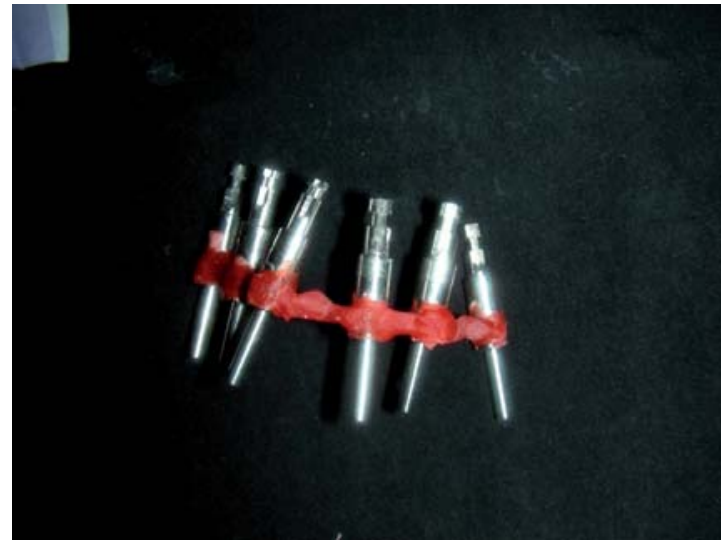

Figura 16. Transfers ferulizados

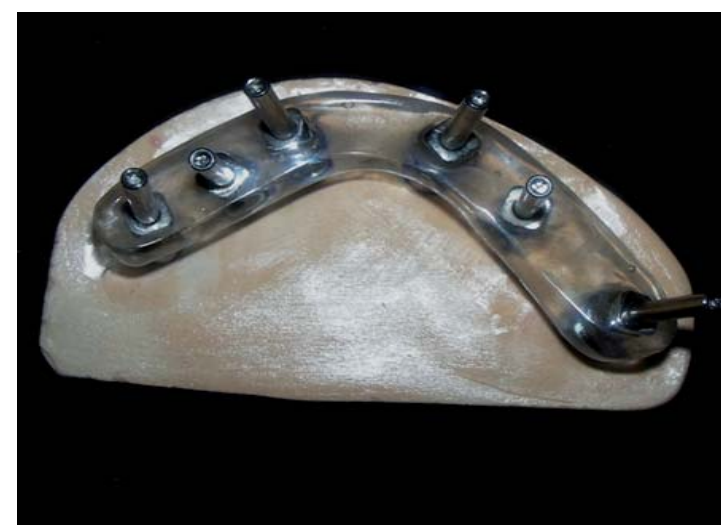

Figura 17. Ferulización de Transfers con acrílico termocurado.

verifico el asentamiento pasivo(Fig 18), si al momento que se colocó el tornillo protésico en un extremo (paso 1) se levantó el otro extremo (paso 2) se procedió a seccionar el acrílico a nivel de la zona donde se levantó y se une nuevamente con acrílico de combustión completa, asegurando así el asentamiento pasivo de la estructura, esta prueba se llama el test de Sheffild, luego se toma la impresión con una cubeta individual abierta a nivel de los implantes, se verificó que la cubeta asiente sin que choque con los transfers, se tomó la impresión con silicona, se colocaron los análogos de pilar y alrededor de ellos se aplicó Gingifast que es una silicona para la reproducción de encía en los modelos de prostodoncia (Figura 19).

En el modelo obtenido se confeccionó una placa base y rodete en el maxilar superior para determinar la dimensión vertical y el contorno, luego se realizó el montaje de los modelos superior e inferior en un articulador semiajustable. Se procedió a enfilar en la placa base dientes poliplanos (Figura 20), este se probó en boca del paciente donde se evaluó la estética

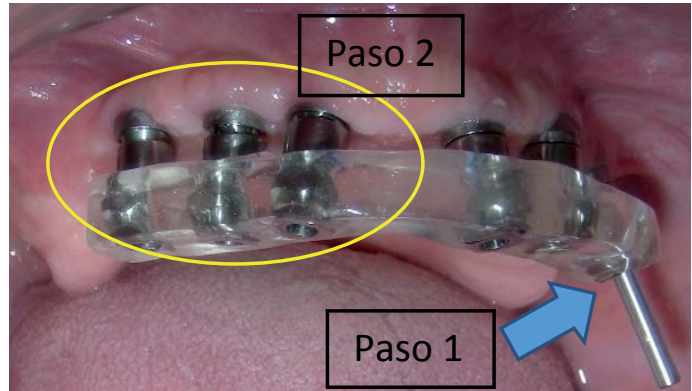

Figura 18. Prueba de Sheffild.

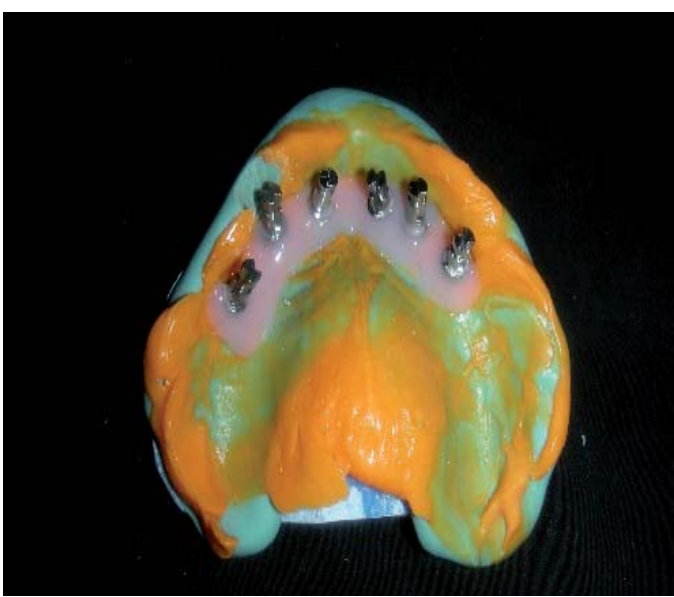

Figura 19. Impresión en cubeta individual.

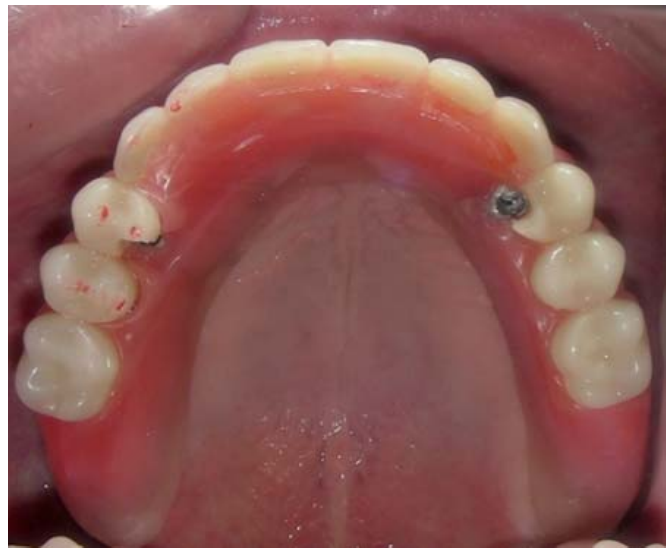

Figura 20. Enfilado de dientes.

y fonética, una vez comprobados estos parámetros, se realizó una llave de silicona vestibular para poder reubicar luego los dientes poliplanos, seenvió al laboratorio para la confección de la estructura colada metálica dentro de los límites del enfilado guiándose con la llave de silicona. Esta estructura es colocada en boca para verificar el asentamiento pasivo del colado sin tensión alguna (Figura 21), y se procede a reubicar los dientes poliplanos considerando la posición del primer enfilado mediante la llave de silicona.

Por último se realiza el acrilizado de la prótesis, es 


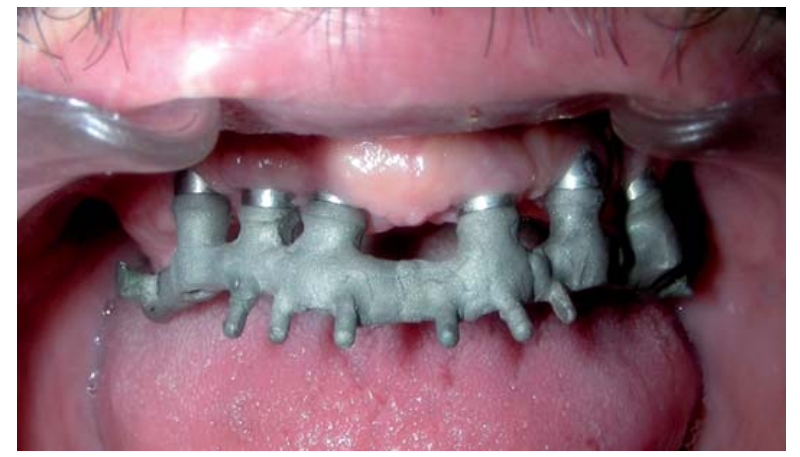

Figura 21. Prueba de estructura colada en boca.

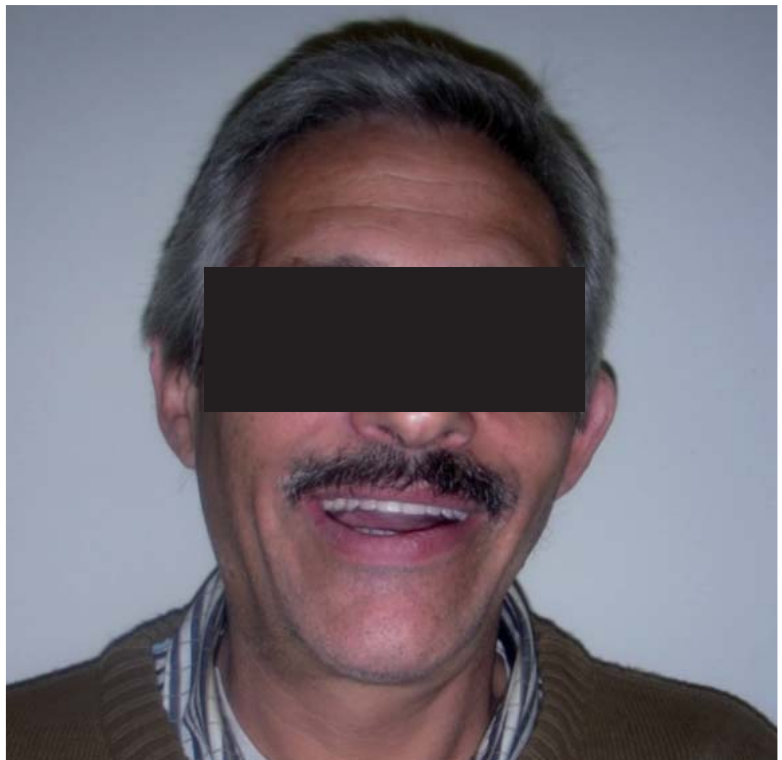

Figura 22. Prótesis hibrida instalada en boca.

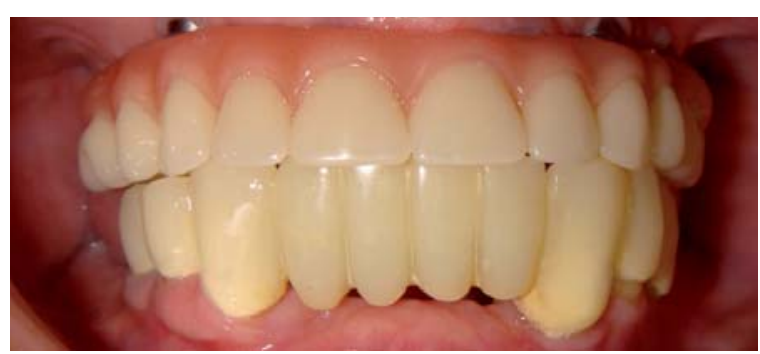

Figura 23. Prótesis hibrida instalada en boca

necesario verificar que haya convexidad de la parte interna y un ligero contacto de la prótesis con la mucosa del reborde para que el paciente pueda realizar una buena higiene de esta zona. Los orificios de acceso a los tornillos (chimenea) se cubrieron con cinta teflón y sobre esta se colocó resina fotopolimerizable. Se ajustó la oclusión, a una mutuamente protegida con función en grupo. Finalmente se instaló una férula de protección (Figuras 22 y 23).

\section{Fase de mantenimiento}

Se realizó primero controles a las 24 hrs, 72 hrs y luego cada mes, pasando los controles clínicos cada 6 meses; se le tomó una radiografía panorámica de control en agosto del 2014, observando que todo está normal (Figura 24).

Se realizó un control clínico en noviembre del 2015, se retiró la prótesis híbrida superior para darle mantenimiento, observando pequeñas inflamaciones alrededor de los pilares pero en buen estado de higiene oral la prótesis híbrida (Figuras 25 y 26).

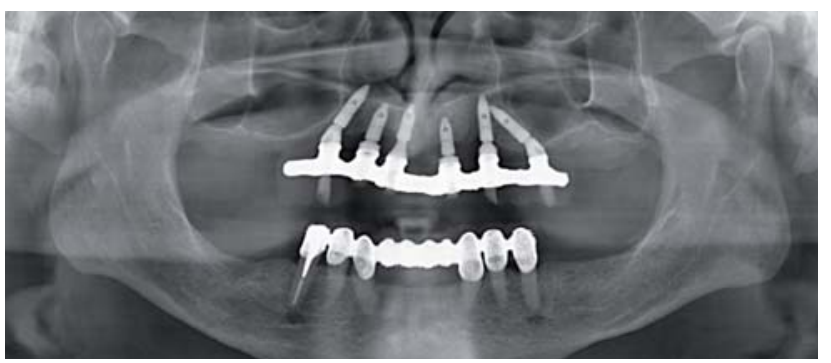

Figura 24. Radiografía panorámica de control tomada el 2014.

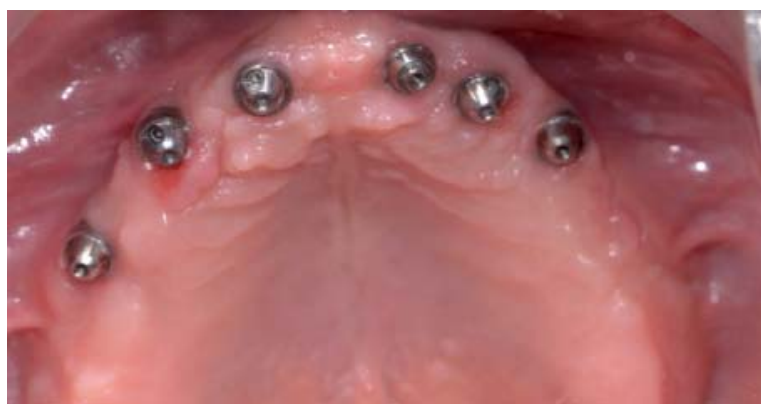

Figura 25. Vista oclusal tomada el 04/11/2015

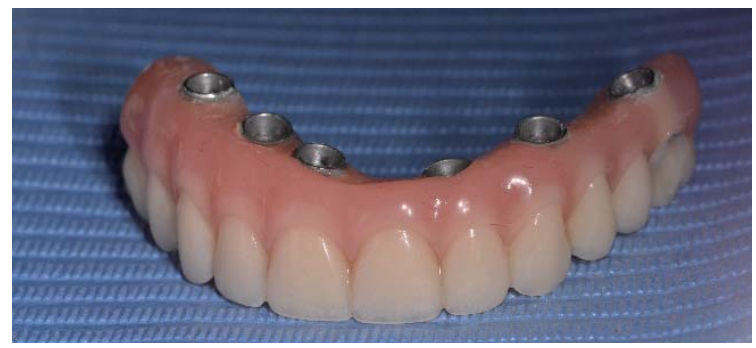

Figura 26. Prótesis híbrida al 04/11/2015 


\section{DISCUSIÓN}

La rehabilitación oral sobre implantes de un paciente edéntulo total empieza con sus expectativas de tratamiento y la correcta evaluación clínica mediante un minucioso examen intraoral y extraoral, un plan de trabajo para ayuda diagnóstica que incluye el estudio por imágenes, que han evolucionado notablemente en los últimos tiempos, modelos con montaje en un articulador tipo semi-ajustable y el protocolo reverso que es primordial para el diseño de la rehabilitación protésica sobre implantes adecuada, pudiendo realizarse prótesis tipo sobredentadura, prótesis hibrida o prótesis fija, esto va a depender del planeamiento que el odontólogo vaya a realizar utilizando parala planificación una guía multifuncional: tomográficaquirúrgica-protésica para la colocación de implantes y el tipo de rehabilitación oral adecuado (9).

La rehabilitación con prótesis hibrida sobre implantes es un tratamiento fijo en maxilares completamente edéntulos en donde el espacio protésico es de $11 \mathrm{~mm}$ o $15 \mathrm{~mm}$ (3), pero en donde la necesidad de un soporte labial por la rehabilitación protésica no es un factor determinante (10).

Las prótesis híbridas implantosoportadas pueden ser un procedimiento de tratamiento alternativo fiable cuando una restauración fija de metal porcelana fundida no satisface los requisitos de un paciente de la estética, la fonética, la higiene bucal y la comodidad oral $(11,12)$.

Brida y cols. proponen un sistema de clasificación de pacientes edéntulos para el uso de prótesis implanto soportada fija, clasificándolo en cuatro tipos según los siguientes factores: a) la cantidad de pérdida de tejido, b) la posición de los dientes anteriores en relación a la ubicación del reborde residual, c) el soporte labial, d) línea de la sonrisa, e) necesidad de material protésico para el color de encía (acrílico rosado). La clase 1, son pacientes que requieren material protésico como acrílico rosado para poder obtener proporción estética de los dientes y contorno óptimo de la prótesis que permita un soporte labial adecuado. La clase 2, son pacientes requerirán acrílico rosado sólo para obtener proporciones dentales estéticas y para el contorno de la prótesis. El soporte labial no es una consideración, ya que la diferencia en la proyección del labio con y sin prótesis es generalmente insignificante. La cla- se 3 , pacientes que no requieren material protésico de color de la encía. Clase 4, son los pacientes que pueden o no requerir acrílico rosado, dependiendo del resultado obtenido después de la intervención quirúrgica (10). Siguiendo esta clasificación el paciente del presente reporte se encuentra dentro la clase 2.

La fabricación de prótesis híbrida en los pacientes con espacio interoclusal adecuado, permite al odontólogo varias ventajas para el aspecto estético. Reemplazo y disminución de soporte de tejido blando en el volumen de la subestructura metálica y en la altura de las coronas en comparación con las prótesis metal porcelana. Además de estas ventajas estéticas, la prótesis híbrida trabaja como un absorbente de fuerzas reduciendo la carga que van sobre los implantes (13). El porcentaje de éxito con el tratamiento de prótesis hibrida implanto soportada es alta, como lo demuestra una revisión sistémica publicada en el 2014, donde se incluyeron 18 estudios para la evaluación, se encontraron altas tasas de supervivencia (5 a 10 años) de $93,3 \%$ a $100 \%$ para las prótesis y de $87,9 \%$ a $100 \%$ para los implantes de soporte (14).

En un estudio retrospectivo donde se evaluó las principales complicaciones después de la rehabilitación con prótesis hibrida implanto soportada, se observó que la principal complicación es la mucositis que afecto el $24 \%$ de los casos, seguido por problemas relaciones con el tornillo protésico en el 13,7\% de los casos estos incluían por ejemplo el desgaste la rosca o la pérdida del mismo, con la misma frecuencia se observaron fractura de los dientes de la prótesis o desprendimiento de este (13,7\%). Estos problemas se relacionaron con un incorrecto registro de dimensión vertical, inadecuada oclusión o falta de ajuste pasivo de la estructura metálica. Otro problema encontrado fue el acceso a los orificios de entrada de los tornillos protésicos (7,8\%) (15).

El cigarrillo contiene más de 4,000 toxinas, incluyendo la nicotina, monóxido de carbono, nitrosaminas, benzenos, aldehídos y cianuro de hidrógeno. La nicotina es un potente vasoconstrictor que reduce el flujo sanguíneo y la entrega de nutrientes donde ocurre la cicatrización. El monóxido de carbono reduce la oxigenación de los eritrocitos, y el cianuro de hidrógeno provoca hipoxia (16).

Rodríguez y col. en un estudio retrospectivo sobre 
la colocación de 1033 implantes oseointegrados y relacionados con complicaciones postoperatorias en pacientes fumadores, encontró pérdida de implantes en pacientes no fumadores con $17,2 \%$, pérdida de implantes en pacientes fumadores con $25,2 \%$, mucositis en fumadores con $11,8 \%$ y en no fumadores con $9,2 \%$, periimplantitis en fumadores con $9,2 \%$ y en no fumadores con 5,3\%. Concluyendo que la pérdida de los implantes es multifactorial debido a los efectos tóxicos del tabaco (17).

Rojas y col presentaron tres casos de pacientes fumadores con prótesis sobre implantes: 2 rehabilitados con prótesis tipo hibrida y uno con coronas individuales de metal porcelana. Se colocaron 44 implantes en los tres pacientes, se perdió un implante en la fase de oseointegración que representa el 2,27\% con un éxito al $97,73 \%$. Lo que demuestra que pueden colocarse implantes en pacientes fumadores, siguiendo el protocolo de la suspensión de fumar una semana antes y una semana después de la cirugía, preservando las paredes vestibulares durante la extracción dentaria, eliminación del tejido de granulación, lograr una estabilidad primaria, relleno de los defectos óseos y cobertura total del implante con cierre primario. (16).

\section{CONCLUSIONES}

La confección de una prótesis hibrida maxilar es una buena opción para rehabilitar maxilares edéntulos en pacientes fumadores, esta debe ser incluida en las opciones de tratamiento cuando se evalúa a un paciente ya que devuelve estética, funcionabilidad, mejora la propiocepción, es fácil de limpiar, menor mantenimiento protésico, puede ser retirada en cualquier momento y realizar reparaciones a un costo muy bajo.

El hábito de fumar no es una contraindicación en el tratamiento con implantes, el éxito de la prótesis dependerá de un adecuado diagnóstico y planificación, además del compromiso por parte de paciente a seguir las instrucciones dadas y también, de la higiene y controles periódicos.

Agradecimientos a la Dra. Lilian Málaga Figueroa docente de la Especialidad de Periodoncia e Implantes, Guillermo Solís Coronado y Jorge Alania Mallqui, alumnos de la Especialidad de Periodoncia e Implantes, y a Dante Alcántara Chávez, alumno de la Especialidad de Rehabilitación Oral.

\section{Correspondencia:}

Martín Quintana del Solar

Correo electrónico: martin.quintana@upch.pe

\section{REFERENCIAS BIBLIOGRAFICAS}

1. Misch CE. Contemporary implant dentistry. 3ra. Edición. St. Louis, Missouri: Mosby Elsevier; 2008.

2. Gonzales J. The evolution of dental materials for hybrid prosthesis. The Open Dentistry Journal. 2014; 8: 85-94.

3. Zarb GA. The longitudinal clinical efficacy of osseointegrated implants a 3 year report, International Journal Oral and Maxillofacial Implants. 1987; 2: 91-100.

4. Cobb G, Metcalf M, Parsell D. An alternate treatment method for a fixed-detachable prosthesis. A clinical report. The Journal of Prosthetic Dentistry. 2003; 89 (3): 239-243.

5. Shibli JA, Piatelli A, Lezzi G. Effect of smoking on early bone healing around oxidized surfaces: a prospective, controlled study in humans jaw. J Periodontol. 2010; 81:575-583.

6. Bain C, Moy P. The association between the failure of dental implants and cigarette smoking. Int J Oral Maxillofac Implants. 1993; 8: 609-615.

7. Grunder U, Gaberthuel T. Boitel N. Evaluating the clinical performance of the osseotite implant: Defining prosthetic predictability. Compend Contin Educ Dent. 1999; 20: 628-640.

8. Bain C. Long-term smoking cessation in dental implant patients. Toronto: $16^{\text {th }}$ Scientific Meeting Academy of Osseointegration; 2001.

9. Balarezo JA. Prótesis sobreimplantes en el edéntulo total: Planificación y elaboración. Lima,Perú: Editorial Savia. 2014;.

10.Brida A, Agar J. A classification system of patients for esthetic fixed implant-supported prostheses in the edentulous maxilla. Compend Contin Educ Dent. 2010;31(5):366-8.

11. Tarnow DP, Emtiaz S, Classi A. Immediate loading of threaded implants at stage 1 surgery in edentulous arches: Ten consecutive case reports with 1- to 5-year data. Int J Oral Maxillofac Implants. 1997;12(3):319-2

12.Real-Osuna J, Almendros-Marqués N, GayEscoda C. Prevalence of complications after the oral rehabilitation with implant-supported hybrid 
prostheses. Med Oral Patol Oral Cir Bucal. 2012;17 (1): 16-21.

13.Qamheya AH, Yeniyol S, Arısan V.Full Mouth Oral Rehabilitation by Maxillary Implant Supported Hybrid Denture Employing a Fiber Reinforced Material Instead of Conventional PMMA. Case Rep Dent. 2015;2015:841745

14. Kwon T, Bain P, Levin L, Systematic review of short (5-10 years) and long-term (10 years or more) survival and success of full-arch fixed dental hybrid prostheses and supporting implants. Journal of Dentistry, 2014; 42 (10): 1228-1241.

15.Egilmez F, Ergun G, Cekic-Nagas I, Bozkaya S. Implant-supported hybrid prosthesis: Conventional treatment method for borderline cases. Eur J Dent. 2015; 9: 442-8.

16. Rojas R, Vargas D, Belmont S. Rehabilitación con implantes osteointegrados en pacientes con tabaquismo como factor de riesgo. Revista Mexicana de Cirugía Bucal y Maxilofacial. 2015;11 (3): 81-90.

17.Rodriguez OF, Figueiredo R, Valmaseda E, Gay C. Postoperative complications in smoking patients treated with implants: a retrospective study. J Oral Maxillofac Surg. 2011; 69: 21522157.

Recibido : 04/04/2015

Aceptado: 10/02/2016 